\title{
Impacts of an Advanced Public Transportation System
}

\section{Demonstration Project}





\section{Impacts of an Advanced Public Transportation System}

\section{Demonstration Project}

Jonathan Levine, Qiang Hong, George Edward Hug, Jr. and Daniel Rodriguez

In 1997, the Ann Arbor (Michigan) Transportation Authority began deploying a set of integrated advanced public transportation system technologies in its vehicles, stations and control center. This paper summarizes selected findings of a multidimensional evaluation of the effects of the system on schedule adherence and transfer time, passenger perception and impacts on drivers. Some improvement in on-time performance was observed in vehicle departures from major transfer points, though not in arrivals. Modest improvements were observed in transfer coordination for routes planned for timed transfers. Deployment of the system did not raise passengers' rating of relevant aspects of their transit experience, but impacts of individual system elements were rated favorably. Focus groups and surveys of drivers revealed a fundamentally favorable attitude toward the deployment of technology, coupled with some concern about limitations it might impose in driver communications and on-the-job creativity. Benefits of the system that were not apparent during the period of the evaluation but may materialize over the longer term include prevention of and response to safety- and security- related incidents, enhanced information for transit planning, and others. 


\section{INTRODUCTION}

In 1997, the Ann Arbor (Michigan) Transportation Authority (AATA) began deploying advanced public transportation systems (APTS) technologies in its fixed route and paratransit operations. The project's concept is the integration of a range of such technologies into a comprehensive system, termed the "Advanced Operating System" (AOS) that would enable "smart buses", "smart travelers," and a "smart operation center" to benefit from timely and coordinated information on critical aspects of transit operation and maintenance.

Part of the APTS demonstration project was a comprehensive evaluation, focusing on a range of project impacts. The evaluation incorporated studies of passengers, drivers, vehicles and system data in a before-and-after evaluation design. This paper provides an overview of three of the studies: a investigation in on-time performance and transferring quality impacts; passenger perceptions; and driver responses to initial system deployment.

Overall, some modest improvements in departure times and transfers were observed, and traveler and driver response indicated positive impacts of AOS on transit service. But given a relatively favorable baseline of performance in the predeployment period, it is not surprising that measurable changes in service outcomes are slight. At the same time it may be that benefits that did not materialize during the initial evaluation period -- for example, in improvements in safety and security -- will manifest themselves over the longer run. Results of these studies may be interpreted as supportive of APTS deployment, though perhaps not immediate and massive retrofitting of all existing systems. Rather, for some systems, an evolutionary approach may be most appropriate under which as transit vehicles, facilities, hardware, and software are replaced, advanced communication and computation are integrated into ordinary processes of design and production. 


\section{Advanced Operating System Concept}

Adapted from AATA's system description. The aspiration of the AATA design of AOS was an integrated deployment of APTS under which synergies between interacting elements of the transit system would be realized. The concept of the interacting "smart bus," "smart operations center" and "smart traveler" guided the development of the system.

\section{"The Smart Bus"}

Central to the AOS concept is the deployment of automatic vehicle location (AVL) technology in order to provide continuous real time data on the location of transit vehicles. Each bus determines its location using global positioning satellite (GPS) technology; differential corrections are broadcast to the vehicles so they can calculate their locations within one or two meters. A Mobile Data Terminal (MDT) in each vehicle stores complete route schedules on an insertable memory card. The GPS system provides accurate time to the vehicles. Buses compare scheduled times and locations with actual locations to determine their schedule adherence. If a bus determines that it is running late, the driver is advised, and if necessary, the onboard computer notifies the Operation Center. The AVL also triggers an outside destination announcement and the internal next-stop signs and announcement. It also integrates location data with automated passenger counters, electronic controlled engine data and ultimately, fare collection.

The AATA network makes use of extensive timed transfers at four major transfer points, including transit centers in downtown Ann Arbor and neighboring Ypsilanti, a shopping center, and the University of Michigan Medical Center. When a bus is running behind schedule, AOS 
enables digital bus-to-bus communications to improve the transfer between buses; the driver of the first bus can send a digital request (including the bus' location) to hold the second bus to ensure that a passenger will not miss a desired transfer. The second bus is authorized to wait up to five minutes in order to facilitate the requested transfer.

Video surveillance is provided on board vehicles for security, as well as to help resolve any claims that may arise from incidents. The paratransit side of AATA operations utilizes AOS as well, with automated routing and scheduling functions. Drivers receive their entire schedules and mark their arrival and departure times with date, time and location information via the MDT as well as all the features above.

\section{"The Smart Operation Center"}

The AATA Operation Center collects and acts upon information provided by the transit vehicle and drivers. Each AATA bus has an 800 megahertz radio and onboard computer. The system minimizes voice transmissions by providing data messages that summarize vehicle status, operating condition, and location. Out-of-tolerance engine conditions such as oil pressure and temperature are reported in real time to the onboard computer, the Operations Center and the Maintenance Department.

Through the use of real time displays of vehicle location and schedule adherence reporting, dispatchers working at the Operation Center can manage the system and assist drivers by inserting overload vehicles in the system or recommending re-routing options. All changes to the route and schedule database are noted and automatically updated.

On board the vehicle, the driver has an onboard emergency system. When encountering a life-threatening situation, the driver covertly alerts the dispatcher, who immediately notes the 
vehicle's location on the system's center map and dials the appropriate agency. The system also allows the dispatcher to open up a central public address system inside the vehicle to monitor the situation. The system also supports responsive reporting of routine, non-life-threatening emergencies, such as passenger inconvenience.

\section{"The Smart Traveler"}

The "smart traveler" is a person informed about his or her transportation options, as well as about current conditions relative to transit use. Inside the bus, next stop announcements, date, time and route are given to passengers utilizing the onboard public address system and a two line LED display. The driver also has the ability to trigger timed and periodic announcements for special events that can be made to support the system. Outside the bus, the current route information is announced to waiting passengers, and the destination signs are changed based upon the location. Later, kiosks will provide real-time bus location information at selected locations; this information will ultimately be provided to travelers at their home or workplace via telephone, cable television or internet.

\section{APTS Planning and Deployment Issues}

A literature on the many dimensions of APTS deployment has been rapidly emerging in the past five years. The most current inventory of APTS deployments is a comprehensive study by Casey (1) of over five hundred transit systems in the United States. APTS functionality and 
deployment is reviewed by Khattak et. al (2) and a comprehensive technological review is provided by Schiavone (3).

In the areas of APTS planning, Hansen, Qureshi and Rydzewski (4) studied processes of APTS adoption and deployment, considering factors leading to decisions to adopt, and developed a framework for understanding APTS benefits; APTS enhances the ability of a system to respond to disturbances in its environment. Levine, Park, Underwood and Wallace (5) studied views of stakeholder groups to an APTS planning and deployment process, and modeled the range of views regarding policy direction for APTS, from views supporting APTS-based service expansion, to a more constrained view focused on improving reliability of existing forms of service.

Rodier, Johnston, Shabazian (6) linked attributes of APTS-related improvements in transit service to regional transportation modeling to estimate consumer welfare changes associated with APTS. While system benefits of congestion and pollution reduction were not forecast, a significant welfare increase for transit travelers emerged from the modeling exercise. The greatest net benefits were associated with deployment of the provision of advanced transit information. Operating cost benefits and increased system utilization were reported by Kihl (7) for the Toronto Transit Commission's automated vehicle location/computer aided dispatch system.

Schweiger and McGraine (8) consider impacts of APTS deployment on transit system employees, and develop recommendations for anticipation and mitigation of personnel related difficulties associated with technology deployment in transit. A number of employee concerns they identify surface in this study's investigation of AATA drivers and dispatchers. 


\section{On-Time and Transfer Performance}

This study seeks to document outcomes of a single APTS deployment. Clearly, results are likely to differ in other public transit systems, especially those varying significantly from the size and service characteristics of the AATA. It is hoped that from evaluations of a range of projects, a clearer picture will emerge regarding the nature and magnitude of APTS outcomes in differing transit environments.

Common to virtually all deployments is a goal of improving transit's schedule adherence. The capacity of APTS to achieve this stems from several functionalities. First, continuous, realtime pacing information to drivers may enable them, in some circumstances, to speed up or slow down between established time points. Ideally this function would facilitate smooth progress through the route and eliminate both the need to wait at time points in order to meet schedules, or (to the extent that this is under drivers' control) the tendency to fall behind. Second, system monitoring by dispatchers can facilitate decisions as sending in another vehicle when needed, or doubling back on a route. In the longer term, the schedule adherence data should provide information to transit planners regarding systematic schedule adherence problems on routes, allowing them to adjust either routes or schedules accordingly. Finally, a non-trivial effect of an automatic vehicle location system is the simple phenomenon of a single clock time throughout the entire system.

Relatedly, systems that plan for timed transfers between routes will seek to enhance the reliablity of the transfer, and to minimize the phenomenon of missed transfers due to late arrival of the bus from which the passenger transfers. Previous to APTS deployment, AATA sought to handle this problem through driver-to-dispatcher coordination; within certain parameters, the dispatcher would call for the second bus to hold for the first. But where transfers are timed, such 
requests will tend to come in rapidly within a very short period of time, and would frequently overwhelm the capacity of the dispatcher to process them. The ability to send digital bus-to-bus transfer requests is the design approach to this problem.

In order to gauge the impact of AOS on both schedule adherence and the timing of transfers within the AATA system, this study developed three matched samples of observations of bus arrivals and departures at the four major transfer points throughout the AATA system. The samples were drawn at randomly selected time periods between March and May for the years 1997 (before APTS deployment), 1998 (early after APTS deployment) and 1999. During 1997 and 1998 observation was carried out on site, whereas after complete deployment of AOS, a schedule adherence dataset was developable through "replaying" sampled routes after the fact at the control center. Approximately one thousand arrivals and a similar number of departures were observed during each of the three years. Arrival and departure times were recorded and compared to time schedules.

Results regarding vehicle arrivals at the four major transfer points are displayed in Figure 1. The figure displays no consistent pattern of improvement between the "before" and the two "after deployment" period. A statistically insignificant two percent decline is observed in the "ideal" time slot: arrivals three minutes early (recorded here as negative numbers) through scheduled arrival time. Overall, early or on-time arrivals remained statistically identical from the first year to the last: 49 percent in 1997 and 50 percent in 1999.

In contrast, the apparent impact of AOS on schedule adherence regarding departures was quite striking (Figure 2). The percent of trips falling in the optimal category (up to three minutes late) jumped from 26 percent in 1997 to 29 percent in 1998 to 44 percent in 1999, an improvement significant with over 99 percent confidence. The bulk of the effect seems to have 
been diversion of trips that previously had left between three and six minutes late to the on-time category. Neverthless, the proportion of very late departures -- greater than six minutes late -did enjoy a modest four percentage point drop.

The reasons for significant improvements in departure schedule adherence without corresponding improvements in arrivals are not obvious from the data, but can be speculated upon. In interviews with drivers (see below) many drivers expressed the feeling that they are continually aware of their adherence or lack of adherence to schedule, even without the pacing function of APTS. The factors leading to late arrivals may well be beyond drivers' or dispatchers' control, including traffic conditions, boarding and securing of wheelchair users, or inclement weather. In contrast, departures from major transfer points tend to be more under the control of the driver. The display of a uniform time systemwide may have a synchronizing impact, in contrast to drivers' reliance on individual wristwatches that vary one from another.

It may be, however, that other phenomena are interacting with this simple synchronization. First, the time display is visible to the passengers, most of whom are aware of the schedule. Thus discomfort at the obviousness of clearly late departures may prompt some drivers to improve schedule adherence to the extent that this is under their control. Secondly, drivers are aware of the potential for electronic observation of bus location by time. The potential of constant surveillance may be a source of inspiration to drivers to improve adherence of their departure time to schedules, even if this means shorter breaks. Finally, some improvement in the schedule adherence of departure times may be attributable to the replacement of voice communications with digital bus-to-bus data transfer. Before the implementation of AOS, a driver of a late bus wishing to request that a bus be held for a transferring passenger would call that information to the dispatcher, who in turn, was to pass it 
along to the driver of the second bus. The problem was that in a timed transfer system, these requests would tend to come in bunches; the dispatcher, unable to cope with the volume of requests would call for a hold on all buses leaving a given transit center, even though perhaps only one or two would receive late transferring passengers. Under AOS, the handling of these requests in direct bus-to-bus digital communications precludes unnecessary waits by buses that are not receiving transferring passengers. This phenomenon would translate into improved adherence to departure time, an outcome observed in figure 2 .

Improvements in departure schedule adherence, combined with the capacity of passengers to request a hold of a bus in order to facilitate a transfer, should combine to reduce transferring time on routes that were scheduled for a timed transfer (defined here as a scheduled wait of six minutes or less). However, the analysis of bus-to-bus transfer time in isolation could be misleading. For example, a zero minute transfer time can arise from both buses running ten minutes late; while better from the passenger's standpoint than a missed transfer, this is hardly to be seen as a desired outcome.

For this reason, this study developed a technique for the concurrent analysis of schedule adherence and transfer time. For all interactions of arriving and departing buses observed during a given period, a transfer time is defined. In particular, this time is the interval between the arrival of any given bus and the arrival time of each other bus on a different, but intersecting route. Transfer times are determined by the next available trip on the relevant route. In this fashion, the thousand arrivals and departures in any period gave rise to roughly six thousand busto-bus transfers, after eliminating from the analysis those route pairs that were not scheduled for a timed transfer. 
In order to analyze the interaction of transfer and on-time performance, each of the busto-bus transfers is represented as a point on a coordinate graph in which the arrival time of the first bus is plotted on the $\mathrm{X}$-axis and the transfer time to the second bus is plotted on the $\mathrm{Y}$ (Figure 3). Observations closest to the origin are clearly desired, as they represent the combination of on-time performance and minimized transfer time. As one moves away from the origin, one observes deterioration in schedule adherence, transfer time, or both.

One might imagine an indifference map plotted on this graph that would, for any individual traveler or transit manager, capture the tradeoff between schedule adherence and transfer time; such an indifference map would convey how much improvement in one would be needed to compensate a deterioration in the other while leaving the observer indifferent to the change. In contrast to the standard economics textbook set of indifference curves, these would be concave to the origin, as the origin represents the optimal point (rather than the usual interpretation, which would be zero consumption). The zones between such curves would represent regions of similar desirability of outcomes.

In order to evaluate change over the three year period, the graphs in Figure 3 define such zones. The precise shape of the zones is a matter for empirical investigation; for the purposes of this study, they were defined as concentric semicircles beginning at the graph's origin. Other specifications are plausible, but the differences in system outcomes would be small. Zone 1, the center of the bull's-eye, is defined as the optimum region, whereas Zone 4, the periphery, represents the least desirable combinations of schedule adherence and transfer time.

The barchart in Figure 3 summarizes the results of the three scatterplots. The proportion of observations falling in Zone 1, the most desirable zone, increase a modest but statistically significant (99 percent confidence) three percentage points between 1997 and 1999. Perhaps 
more significantly, the percent of transfers in the worst category -- zone 4 -- declined six percentage points over the period.

It should be noted that these results are subject to external sources of variance, such as variations in traffic conditions that are both beyond the control of the system and external to the analysis. But overall, while massive improvements in schedule adherence systemwide did not materialize -- in part due to a reasonably high baseline -- in departures and in transferring time, some significant improvements were apparent.

The unit of analysis in this section is the bus itself; thus, bus-to-bus transfer data were monitored regardless of the presence or absence of transferring passengers. The extent to which schedule adherence, transfer time and other AOS related outcomes not associated with timing are perceived by passengers is considered next.

\section{Customer Response}

In order to gauge customer perception of and response to AOS-associated changes, an on-board survey was conducted of AATA's fixed route bus passengers during three consecutive springs. Opinions and attitudes of passengers were first collected in the spring of 1997, before AATA introduced the AOS. The second set of data was collected in the spring of 1998 during a period in which AATA hand begun operating the AOS, although full integration of all parts of the AOS were not implemented by the end of the survey period. By the third survey period in the spring of 1999, the AOS achieved almost full operational status.

Routes were sampled randomly within a stratified sample design; for each route in the system five trips were sampled randomly during the study period. Written survey questionnaires 
were distributed to all passengers. Approximately 110 to 120 trips were sampled in each year, with an average of ten responses collected per trip.

Impacts of AOS on passenger perceptions of AATA service area mixed. When asked to rank different aspects of AATA service, passengers offered ratings that were quite high in the base year, tending to over four out of five possible points on average. This high base may explain in part why the values did not improve, an even deteriorated between 1997 and 1999 (Table 1). If anything, those elements that should be directly affected by AOS, including transfers and on-time performance, seemed to suffer the worst decline in ratings.

While overall rankings of rider satisfaction show no positive impact, when questions directed the respondents to specific elements of AOS, a more optimistic picture emerges. Table 2 reports results in 1999 to the following question: "Over the past year, AATA has made some technological changes. Please indicate what effect you think they've had on the quality of service that you receive." Ratings ranged from 1 ("made a lot worse") to 5 ("made a lot better.") Mean scores were consistently high, interestingly, with the visual time display receiving the highest ratings. Other service that passengers indicated had a particularly great impact included voice announcements of stops, and the capacity of automated transfer requests.

Inference from these mixed results is a matter of interpretation. Ideally the impact of an APTS deployment such as this one would so improve the transit experience that general satisfaction ratings would increase; this clearly did not happen in the three samples. But when respondents were asked to focus on individual AOS elements and the impact that they had on their service, a significantly more positive response emerged. Thus the favorable impact that travelers perceived regarding individual system elements is apparently too modest to alter perceptions of transit level of service overall. 


\section{Driver and Dispatcher Response}

Success of a system like AOS is dependent in large measure on its effect on the individuals who come in closest contact with the technology, and their perceptions of its contribution to their ability to do the job. For this reason, this study examines perceptions of drivers and dispatcher regarding AOS. This was accomplished in two separate investgations: a series of focus groups, and a driver survey.

In late September 1997 a series of four focus groups was conducted with AATA drivers, driver/trainers and dispatchers regarding the AATA Advanced Operating System (AOS) at its early phases of deployment. The purpose of the focus groups was to assess perceptions regarding the hoped-for AOS benefits, any impediments to realizing those benefits, and any perceived disadvantages of AOS from the standpoint of the driver or dispatcher.

\section{Perceived Benefits of AOS}

Overall, participating individuals saw considerable potential benefit emerging from the AOS. A regularly mentioned benefit was that of enhanced transferring; drivers express a keen interested in customer satisfaction, and see effective transferring between transit vehicles as key to achieving this. Drivers noted that the previous system frequently became too busy to notify connecting buses of transferring passengers when necessary; indicated satisfaction at the ability to send notifications of transfers directly rather than through dispatchers; and appreciated the ability to leave a digital message which allowed communications even when one party was not available at the moment.

Another frequently mentioned benefit is that of removing the driver's burden of 
addressing passengers directly to announce stops or to put a stop to unwanted behavior, as well as the requirement to change and update signs. Apart from the potential for relief from repetitive tasks, drivers appeared eager to avoid injecting their personality into certain on-board situations. Automated voice announcements were not universally perceived as an unadulterated benefit, as other drivers were less tolerant of the constant presence of the recorded voice.

The benefits of camera surveillance were also seen to be considerable. These ranged from providing evidence in case of disputes to enhancing on-board security, solving accident claims, maintaining among teenage riders, and helping prevent littering, dirtying or damage to the buses interior.

A controversial feature of the AOS was that of the pacing information informing the driver of the bus' late or early status. A number of drivers felt that the information was unneeded, indicating that the driver is constantly aware of his or her location and timing, even without the external information. Moreover, some drivers saw disadvantages to the pacing information. Since passengers are able to see the information on the bus' mobile data terminal, drivers feared that the "late" display might provoke some hostility. Despite these hesitations about the pacing function, drivers allowed that there were potential benefits in certain situations, including trips by new drivers, or by drivers taking new routes.

For longer term service planning, drivers saw benefits to the comprehensive data collection of the AOS. Some felt that the system would help back up their claims regarding certain routes and schedules that are particularly difficulty to maintain. Dispatchers saw great potential benefit to the system, in many cases more than the benefit perceived by drivers. They indicated that without AOS they feel sometimes as if they are "working in the dark" and hence unable to do their job. By identifying locations of all vehicles in real time, AOS promises to 
allow them to act in a more proactive and professional dispatching capacity rather than in constant "firefighting" mode.

In addition, dispatchers anticipate the ability to provide accurate information about system delays. Snow days, for example, generate numerous inquiries regarding delays on particular lines, but only general information can be provided. AOS promises to afford dispatchers the ability to provide updated and specific information about travel conditions under such circumstances.

\section{AOS Design}

Drivers were asked about details of the design of the AOS that might facilitate or impede the hoped-for benefits described above. Drivers generally accepted the design and interface of the system, indicating that it fit in relatively well with their working style. Nonetheless the substitution of digital for voice communications with dispatchers was seen as having its disadvantages. The system significantly constrains the voice communications that are possible between driver and dispatcher, and element that is found frustrating in certain situations.

Similarly, while the automated bus-to-bus transfer request was a greatly appreciated function, drivers felt that drivers' inability to communicate directly with other drivers impeded their functioning in certain situations. For example, drivers may creatively facilitate transfers when they know that they are running too late to meet at the established transfer locations, and immediate driver-to-driver communication is seen as critical to this capability. Since these "onthe-fly" transfers are not preprogrammed, they require direct voice connections between buses, and drivers regret that the system does not afford them that opportunity.

Paratransit deployment raised particular concerns. For example, flexibility and 
responsiveness in voice communications is seen as important for dealing with passengers who fail to appear for a paratransit pickup; digital communications are seen as less appropriate in this situation. Relatedly, automated tracking of passengers who fail to show up for their rides was seen as desireable in order to ensure an automatic deletion of their return trip.

There was general acknowledgment that AOS led to efficiencies in paratransit pickup and dropoff. Yet there was concern that this may have come at the expense of some passengers being forced to ride around too long as others were being accomodated. Excess length of trip for some passengers was of particular concerns with people with disabilities or medical conditions. Relatedly, there was some feeling that paratransit routing under AOS was particularly hectic for the driver: With the automatic routing and scheduling, "you run around like a chicken with its head cut off," one driver indicated.

\section{Perceived Disadvantages of AOS}

For some drivers, the deployment of advanced technology seems primarily to represent a relief of burden of tedious tasks, freeing them to concentrate on more creative aspects of their work. For others, the introduction of technology is seen as imposing unnecessary structure and rigidity. Drivers' prognosis regarding the desirability of the AOS is largely determined by where their perceptions fall along this continuum.

For example, while video monitoring was welcomed by most drivers for its potential in safety and dispute resolution, one driver saw potential for crimping an interpersonal style:

"I make acquaintances of regular passengers; with a monitoring system there is a negative effect on interaction with other passengers. As automation goes up, the human 
touch decreases."

Similarly, concerns were expressed regarding the potential for technology to create dependency in drivers, and an inability to think on their own:

"I do not want to see everyone getting to a mode of defaulting to the system. They should use their head in thinking about ways of solving problems. It fosters reliance on the system."

Finally, there is some perception that drivers had been shortchanged in the overall AOS deal relative to customers and management. Where management acquires better control over equipment and the system as a whole, and customers enjoy improved information services, some drivers felt that benefits accruing to them are slight indeed.

\section{AOS and Overall Job Satisfaction}

Job satisfaction impacts were similarly affected by perceptions of technology's influence on one's individual working style. Some drivers anticipated rise in status associated with the technological skill level of the new job description; operating a high technology vehicle is seen as being more than a bus driver. In contrast, others expressed concerns regarding potential intrusiveness of the system:

"Every driver speeds. At some point you bend the rules, and then under AOS you have problems with liabilities. It is always against the driver." 
Perhaps the most pervasive attitude was a combination of acceptance and resignation:

"As technology progresses, you could be with it or without it, but you have to keep up.

The system isn't very intrusive. This is the direction the job is going. There is no choice, you have to move on and adjust."

Drivers indicated that their job satisfaction stems largely from their ability to interact with passengers in a positive fashion, to use their creativity to serve passenger needs and have autonomy and responsibility over their working day as "captains of their ships." Where AOS is seen as serving these goals, it tends to engender satisfaction among drivers; where it interferes with them, drivers may be resentful of its use.

\section{Driver Survey}

In order to quantify issues raised in the focus groups, a survey was conducted of AATA's motor coach operators. The purpose of the survey was to seek drivers' views on the various aspects of AOS, including the automatic vehicle location systems, the mobile data terminals (MDT) and the video camera surveillance system. The written survey questionnaire was distributed to all 103 AATA Motor Coach Operators, with a request to respond anonymously. The overall response rate was 63 percent, or 65 responses out of 103 surveys distributed.

\section{Survey Findings}

The results in table 3 seem to incorporate many of the disparate views that drivers hold regarding 
the introduction of advanced technology. Communications are seen as critical to effective operations. While the mobile data terminals can reduce the ambiguity of many messages, the drivers tend to feel that the lack of open voice communications burdens communications with dispatchers and other drivers. Especially popular among drivers are the burden-relieving functions of automated voice announcements and automated sign changing.

Pacing information received a relatively strong endorsement in the survey, despite the somewhat negative views expressed in the focus groups. Importantly, the judgment regarding AOS overall was positive: only fourteen percent of drivers agreed with the statement, "I like driving without AOS better than with it."

Analysis of subgroups reveals noticeable differences in views of AOS between men and women, and notably between veteran Motor Coach Operators and more novice drivers. In general, drivers with fewer years of experience are more satisfied with AOS than more senior drivers (Table 4). It appears that those whose driving experience is nearly exclusively with AOS lack the resistance of some of the veteran operators. In the area of safety, female drivers' perceptions differ markedly from those of their male counterparts. The safety-promoting effects of video and audio surveillance, as well as the knowledge that the precise location of the vehicle is always known, apparently combine to create an improved sense of safety among female drivers.

\section{CONCLUSION}

An evaluation of a system such as AOS over a relatively short time period necessarily focuses on certain outcomes, and tends to exclude others. The modest results described here should be viewed as a starting point for description of the potential improvements of advanced public 
transportation systems to transit passengers and operators. Potential less tangible or more long term benefits include such items as:

Maintenance: Constant electronic monitoring for potential mechanical problems such as low coolant or oil should save both drivers' labor (since the computers will provide all mechanical information) and may in the longer term prevent of mechanical problems and interruption of bus service. Increased mechanical reliance should also reduce the need for road calls and should improve customer confidence.

Costs of Legal Claims: Video monitoring, installed as a component of AOS, can not only capture the activities inside the bus, but also some activities outside of the vehicles. In the longer term it is expected that will help to resolve complaints and legal claims, and may ultimately reduce cost associated with such disputes.

Data Quality for System Planning: Constant monitoring of vehicle location and automated passenger counting provides a wealth of data used in service planning. In general, the data are not currently organized into the kind of reports required by transit planners and managers, so this benefit remains elusive for the moment. Future generations of APTS hardware and software will undoubtedly benefit from early experiences such as the one described here in developing data in forms useful to transit end users.

Improvements directly measured through the studies reported here represent relatively marginal changes in the level of service that transit is currently able to offer its users. There are a number of reasons why unambiguous service benefits were not documentable by this study. First was a limitation of study design. The ideal study design would be one where half the fleet was outfitted with AOS and the other half was not; this would have helped eliminate the effects of significant extraneous sources of variation, such as road construction. Unfortunately, this 
design was not possible given the imperatives of systemwide AOS deployment. Second, the process of AOS installation was considerably longer than initially anticipated. As a consequence, this study is best interpreted as a "before" and "during" design, as opposed to its original conception of a "before" and "after" design. Finally, some key elements in passenger information were still not deployed as of this study, and were thus not reflected in the data collected.

These results alone do not seem to justify massive and immediate retrofitting of all transit systems with integrated advanced public transportation systems. At the same time, they are suggestive of longer term benefits that would be realized through the deployment of advanced technology in transit. One possible approach would be an evolutionary strategy: as vehicles and systems are replaced, new ones are deployed that incorporate many of the capacities described here. If based on consistent standards, this approach promises, over time, to enable public transit to reap the significant operational and level-of-service benefits of rapid technological improvement.

\section{REFERENCES}

1. R. F. Casey. Advanced Public Transportation Systems Deployment in the United States. Volpe National Transportation Systems Center; Federal Transit Administration. 1999.

2. Khattak, Asad, et. al. Advanced Public Transportation Systems: A Taxonomy, Commercial Availability And Deployment. California PATH Program, Institute of Transportation Studies, University of California at Berkeley, 1997. 
3. J. J. Schiavone. Understanding and Applying Advanced On-Board Bus Electronics. TCRP Report 43, National Academy Press, Washington, D.C., 1999.

4. M. Hansen, M. Qureshi, D. Rydzewski. Improving Transit Performance with Advanced Public Transportation System Technologies. California University, Berkeley, Institute of Transportation Studies, August 1994, p. 121.

5. J. Levine, S. Park, S. E. Underwood, and R. R. Wallace. Stakeholder Preferences in Advanced Public Transportation System Planning. Journal of Public Transportation, 1999, 2(2) p. 25-45.

6. C. J. Rodier, R. A. Johnston, and D. R. Shabazian. Evaluation of Advanced Transit Alternatives Using Consumer Welfare. Transportation Research C: Emerging Technologies, February 1998, p. 141-156.

7. Kihl, M., and D. Shinn. "Improving Interbus Transfer with Automatic Vehicle Location." August 1993 (year one report).

8. C. L. Schweiger and J. Mcgrane. Deployment of Technology for Paratransit: What Are the Effects on Employees? Mobility for Everyone 4th World Congress on Intelligent Transport Systems, October 1997, Berlin. 


\section{AUTHOR AFFILIATION}

Jonathan Levine

Qiang Hong

George Edward Hug, Jr.

Daniel Rodriguez

Address and affiliation for all

Urban and Regional Planning Program

A. Alfred Taubman College of Architecture and Urban Planning 2000 Bonisteel Blvd.

The University of Michigan

Ann Arbor, MI 48109-2069

734-763-0039 